\title{
Single and Multiple Longitudinal Wavelength Generation in Green Diode Laser
}

\author{
M. Hosne M. Shamim, Tien K. Ng, Senior Member, IEEE, Boon S. Ooi, Senior Member, IEEE, and \\ Mohammed Z. M. Khan, Senior Member, IEEE
}

\begin{abstract}
Single and multiple wavelength laser systems are presented that employ self-injection locked InGaN/GaN green laser diode in an external cavity configuration with a partially reflective mirror. A stable and simultaneous locking of up to 4 longitudinal Fabry-Perot modes of the system cavity is demonstrated with appreciable signal-to-noise-ratio (SNR) of $\sim 13$ dB and average mode linewidth of $\sim 150 \mathrm{pm}$. The multiwavelength spectrum exhibited a flat-top emission with nearly equal power distribution among the modes and an analogous mode spacing of $\sim 0.5 \mathrm{~nm}$. This first demonstration of multiwavelength generation source is highly attractive in a multitude of cross-disciplinary field applications besides asserting the prospects of narrow wavelength spaced multiplexed visible light communication. Moreover, an extended two-stage self-injection locked near single wavelength visible laser system is also presented. An ultra-narrow linewidth of $\sim 34 \mathrm{pm}$ is realized at $525.05 \mathrm{~nm}$ locked wavelength from this innovative system, with $\sim 20 \mathrm{~dB}$ side-mode-suppression-ratio (SMSR); thus signifying a paradigm shift towards semiconductor lasers for near single lasing wavelength generation, which is presently dominated by other kinds of laser technologies.
\end{abstract}

Index Terms - External cavity diode laser, self-injection locking, visible multi-wavelength laser, single frequency laser.

\section{INTRODUCTION}

$\mathrm{V}$ ISIBLE single/multiple longitudinal wavelength sources are invaluable for various multi-disciplinary field applications [1]. In particular, they are indispensable sources in optical communications, medical diagnostics, optical cloaking, metrology, precision spectroscopy, bio-sensing, microwave photonics, etc. [2]. In literature, visible coherent

Manuscript received 31 January 2019. Authors acknowledge the support from King Abdulaziz City for Science and Technology (KACST), (grant nos. EE2381 and KACST TIC R2-FP- 008); partial support from King Abdullah University of Science and Technology (KAUST) baseline funding (grant nos. BAS/1/1614-01-01, KCR/1/2081-01-01, and GEN/1/6607-01-01); and KAUST-KFUPM Special Initiative (KKI) Program (REP/1/2878-01-01).

M. H. M. Shamim was with the Optoelectronic Research Laboratory, Department of Electrical Engineering, King Fahd University of Petroleum and Minerals (KFUPM), Dhahran 31261, Saudi Arabia. He is currently with the Department of Electrical and Computer Engineering, McGill University, Canada. (e-mail: hosne.shamim@mail.mcgill.ca).

T. K. Ng, and B. S. Ooi are with Photonics Laboratory, Computer, Electrical and Mathematical Sciences and Engineering (CEMSE) division, King Abdullah University of Science \& Technology (KAUST), Thuwal 23955-6900, Saudi Arabia (e-mail: tienkhee.ng@kaust.edu.sa; boon.ooi@kaust.edu.sa).

M. Z. M. Khan is with Optoelectronic Research Laboratory, Department of Electrical Engineering, King Fahd University of Petroleum \& Minerals (KFUPM), Dhahran 31261, Saudi Arabia (corresponding author: e-mail: zahedmk@kfupm.edu.sa). single and multiple longitudinal wavelength emissions are demonstrated on external cavity diode laser (ECDL) system and semiconductor gain medium - optical fiber configuration, respectively. While the former technology employs a wavelength grating filtering mechanism for single wavelength selection, the latter technique exploits various non-linear phenomena to realize a coherent frequency comb or multiwavelength generation. In the following, we briefly review these technologies.

ECDL system in Littrow configuration is one such system that has been used often in the visible wavelengths to realize single longitudinal wavelength emission as well as tunable lasers. For instance, Chen et al. demonstrated a single wavelength operation in green emission wavelength with $\sim 10$ $\mathrm{MHz}$ linewidth [3]. Subsequently, a more stable system at $\sim 522 \mathrm{~nm}$ using iodine was demonstrated; however, at the cost of broader linewidth, in $\mathrm{GHz}$ range [4]. Moreover, green tunable laser in the range of $509-518 \mathrm{~nm}$ with $\sim 17 \mathrm{~dB}$ amplified spontaneous emission (ASE) suppression was also demonstrated using a similar configuration [5]. However, the Littrow configuration ECDL system suffers from high loss and alignment issues [6]. Very recently, a blue GaN distributedfeedback laser diodes have been reported using high order notch gratings to obtain single wavelength emission with a narrow linewidth of $6.5 \mathrm{pm}$ and $35 \mathrm{~dB}$ side mode suppression ratio (SMSR) [2].

Incoherent and coherent multiple longitudinal wavelength emission in the form of frequency comb generation has been reported in AlN/SiN micro-resonators and optical fibers, respectively, employing non-linear effects. For instance, a highly coherent visible optical frequency comb spanning from $500 \mathrm{~nm}$ to $1200 \mathrm{~nm}$ was demonstrated from a $1550 \mathrm{~nm}$ Erdoped fiber laser using frequency double conversion [7]. 14 comb lines in visible wavelengths were demonstrated in a dispersion engineered fiber using four-wave mixing, phase matched at $765 \mathrm{~nm}$ [8]. However, to the authors' knowledge, multiple longitudinal wavelength generation from a visible diode laser has not been reported. Such a compact system has already attracted attention in the near infrared region $(\sim 1300$ $1630 \mathrm{~nm}$ ) with promising demonstrations in the microwave and terahertz optical beat frequency generation, indoor data center communication, and wavelength division multiplexed passive optical networks [9]-[12]. In this case, various nanostructures viz. quantum well [13], quantum dash [14][16], and quantum dot [17], [18] based lasers have been 
engaged. Hence, a compact coherent multiple wavelength visible laser diode with narrow and uniform wavelength spacing is of paramount importance to facilitate high capacity wavelength division multiplexed visible light communication (WDM-VLC) system, which is considered as a viable last mile-access network infrastructure. Moreover, unlike the recent demonstration of WDM-VLC [19]-[22] links wherein wide wavelength spaced red-blue-green colors are employed as sub-carriers, such a narrow-spaced multi-wavelength visible laser source based network architecture would enable optimization of the available optical bandwidth.

Very recently, we proposed a new-class of wavelength filter-less ECDL system employing assisting self-injection locking scheme. By implicitly inheriting the distinct locking characteristics, we demonstrated narrow lasing emission linewidths of $70-120 \mathrm{pm}$ on red, blue, and green color laser diodes [23]. Moreover, a wavelength tunability in the range of $\sim 521-528 \mathrm{~nm}$ with high output power was also realized employing this configuration.

In this work, we further substantiate the potential of this new class of ECDL system as a viable solution for realizing highperformance visible laser diodes. Firstly, by employing a firstof-its-kind extended three-cavity ECDL system with two-stage self-injection locking, we demonstrate a single-wavelength operation at $525.05 \mathrm{~nm}$ with $\sim 34 \mathrm{pm}$ linewidth and $\sim 20 \mathrm{~dB}$ SMSR. Secondly, a coherent, narrow-spaced, multiple longitudinal wavelength generation is realized with a two cavity self-injection locked ECDL system. By simultaneously locking up to 4 Fabry-Perot (FP) modes with $\sim 0.5 \mathrm{~nm}$ spacing, a coherent frequency comb source is realized for the first time, to our knowledge, on a semiconductor diode laser based system in the visible wavelength. Mean linewidths of $\sim 150 \mathrm{pm}$ with an average $\sim 15 \mathrm{~dB}$ SMSR is achieved with comparable peak and integrated powers, and stable operation.

\section{EXPERIMENTAL SETUP AND BASIC LASER DIODE CHARACTERIZATION}

The experimental setup for the multiple and single wavelength laser system is shown in Fig. 1 (a) and (b), respectively. We have used a commercially available single spatial mode $\mathrm{InGaN} / \mathrm{GaN}$ green laser diode LD (Thorlabs L520P50) as a source whose emission wavelength is in the range of $520-529 \mathrm{~nm}$. The green laser beam is allowed to pass through an aspheric lens $\mathrm{L}_{1}$ (Thorlabs, A110TM-A) and after being collimated, $92 \%$ of the light beam traveled through a 92:8 pellicle beam splitter (BS, Thorlabs, BP108). An external cavity resonator is formed between the front facet of the laser diode and the partial reflector $\mathrm{R}_{1}$ (polka dot beam splitter from Edmund optics) with $30 \%$ reflectivity. $\mathrm{R}_{1}$ is installed on a manual kinematic mount (Thorlabs, KM100) and single-axis translational stage, at the other end of the optical axis and a distance $d_{1}=26 \mathrm{~cm}$. Kinematic mounts enable slightly angular as well as linear tuning of the reflected beam and hence of the tuning of external cavity length is done. The other $8 \%$ reflected the light beam from the BS was coupled into an optical fiber through a biconvex lens $\mathrm{L}_{2}$ (Thorlabs, LB1471-A-ML) of focal length $50 \mathrm{~mm}$, for spectral analysis utilizing an optical spectrum analyzer (OSA, Yokogawa AQ6373B with $0.02 \mathrm{~nm}$ resolution). It is worth mentioning at this instant that the $70 \%$ of the transmitted power through $\mathrm{R}_{1}$ appears as the output of the system that is $\sim 65 \%$ of the laser diode optical power, thus reducing the system loss considerably. Besides, collection of the output power from the back facet of the laser diode would negate the loss of the power entirely. (a)

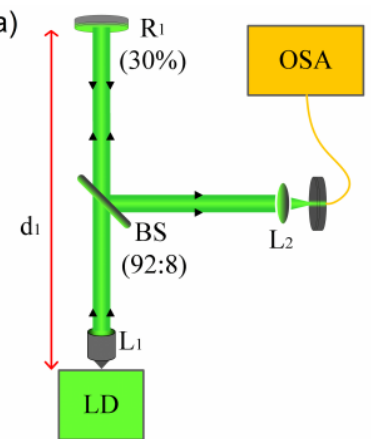

(b)

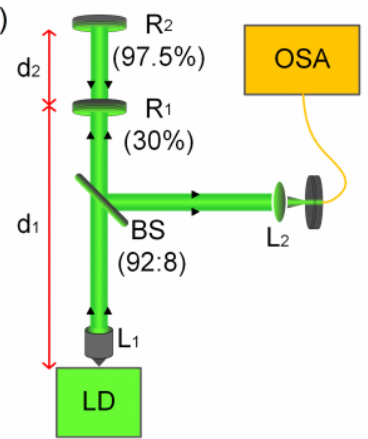

Fig. 1 Block diagrams of (a) single stage (for tunable laser and multiwavelength generation) and (b) two-stage (near single wavelength generation) self-injection locked laser system employing $\mathrm{InGaN} / \mathrm{GaN}$ green laser diode.

Fig 2. (a) shows the lasing spectrums of the $I n G a N / G a N$ laser diode at 40,90 , and $160 \mathrm{~mA}$ injection currents spanning from just above the threshold to the maximum injection before the rollover with output power exceeding $10 \mathrm{~mW}$. The $-3 \mathrm{~dB}$ linewidths of the spectrums broaden gradually and redshifts, a legacy of bandgap shrinkage because of junction temperature rise due to an extreme surge of carriers. The threshold current of the laser diode is measured to be 37 and $47 \mathrm{~mA}$ at 20 and $40^{\circ} \mathrm{C}$, respectively, as observed from the $L-i-V$ curves in Fig. 2 (b). Moreover, at higher operating temperature, lower bias voltage and the emitted optical power with degraded slope efficiency are apparent across all the injection currents, which is attributed to the temperature-driven decrease of the internal quantum efficiency and the increase of the internal loss [25].
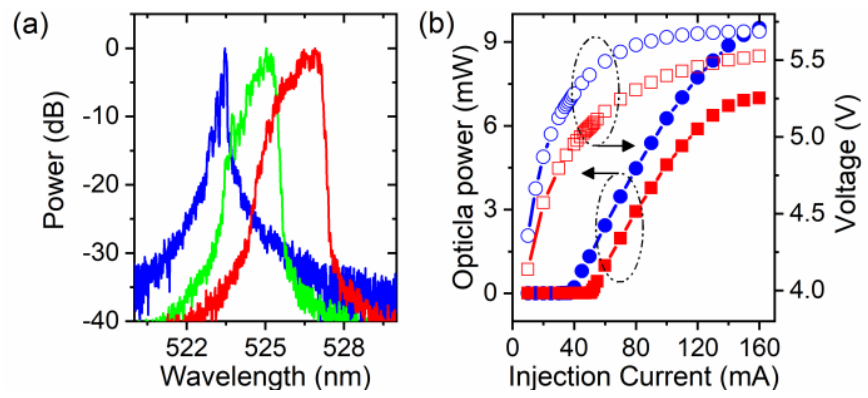

Fig. 2 (a) The free running optical spectrums of the laser diode at 40 (blue), 90 (green), 160 (red) $\mathrm{mA}$ recorded at $20^{\circ} \mathrm{C}$ temperature. (b) $L-i-V$ characteristics of the free running laser diode at $20^{\circ} \mathrm{C}$ (blue) and $40^{\circ} \mathrm{C}$ (red) temperature, measured after $\mathrm{L}_{2}$. 


\section{NARROW LINEWIDTH LASER}

\section{A. Tunable Wavelength Generation}

A two-cavity system as shown in Fig. 1 (a) comprising the laser diode cavity and the external cavity allows longitudinal modes of different free spectral ranges. Locking of a specific wavelengths using self-injection locking scheme takes place if the free spectral range (FSR) of the laser cavity is an integer multiple of that of the external cavity; in other word, roundtrip phase matching condition should be satisfied. This phenomenon follows the relation between FSR and the cavity length as shown in (1).

$$
\Delta \lambda_{F S R}=\lambda^{2} / 2 n L
$$

Here, $L$ represents the length of the cavities that are $900 \mu \mathrm{m}$ and $26 \mathrm{~cm}\left(\mathrm{~d}_{1}\right)$ for the laser and external cavity, respectively. Hence, by careful tuning of the external cavity length via slight movement of tilt and linear stages, we were able to selfinjection lock various modes of the laser diode and tune them discretely, as shown in Fig. 3(a). Near single longitudinal modes at different wavelengths in the range of 521.10 to $527.63 \mathrm{~nm}$ is apparent from the figure with a cumulative tunability of $6.53 \mathrm{~nm}$ that is obtained from three injection currents 36,95 , and $160 \mathrm{~mA}$. The performance of the system is depicted in Fig. 3 (b), which plots the achieved SMSR and the lasing linewidth (full-width-at-half-maximum) across the tuning window. SMSR as high as $\sim 20 \mathrm{~dB}$ is observed at the lower injection current and later saturates to $\sim 10 \mathrm{~dB}$ at high current injections. On the other hand, a steady linewidth of 100 $\pm 20 \mathrm{pm}$ is measured in the entire wavelength tuning range and is found to be independent of the injection current, thanks to the efficient locking at that particular wavelength. Moreover, the effect of temperature on the system demonstrated a robust and stable tunable laser system.
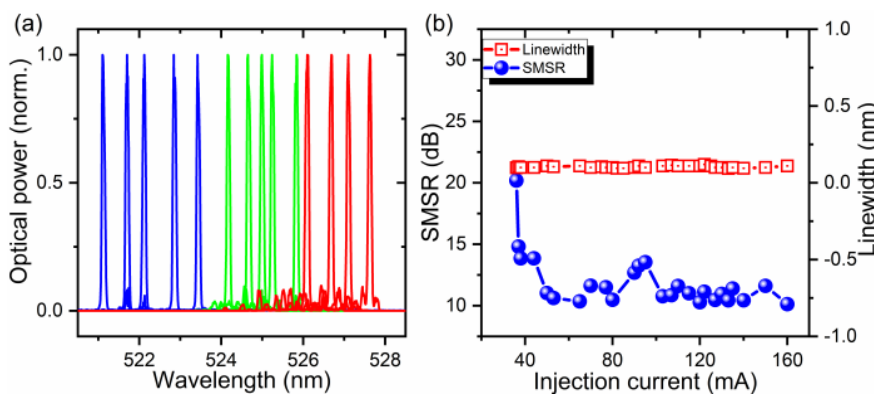

Fig. 3 (a) Overlapped lasing spectrums showing $6.53 \mathrm{~nm}$ discrete tunability of the system at 36,95 , and $160 \mathrm{~mA}$ injection current as shown in blue, green, and red color respectively. (b) SMSR and linewidth of the locked modes at various current injections as shown in blue spheres and red squares with a dot inside, respectively.

\section{B. Single Wavelength Generation}

Fig. 1 (b) shows an extended three ECDL system to realize a single wavelength lasing operation. An additional reflector $\mathrm{R}_{2}$ of reflectivity $97.5 \%$ (Thorlabs, PF10-03-P01) is placed after
$R_{1}$ along the optical axis such that the distance $d_{1}+d_{2}=26 \mathrm{~cm}$. In this case, $R_{1}$ is shifted closer towards the laser diode front facet and placed at a distance of $\mathrm{d}_{1}=16 \mathrm{~cm}$. Hence, the composite cavity of the system now constitutes three FP resonators that include two external cavities of length $d_{1}$ and $\mathrm{d}_{2}$. In this case, the usable output beam would be the reflected $8 \%$ from the BS that is used for diagnostic purpose. Since the reflected laser beam from two reflectors, $R_{1}$ and $R_{2}$, are optically feedback into the laser active region, the configuration is termed as two-stage self-injection locked external cavity laser system, and the first to be proposed and demonstrated here, to the authors' knowledge. A similar resonance frequency and phase matching conditions dictate this system, however, would be more stringent since here a particular mode from all the sustaining modes in the composite cavity should satisfy the phase matching condition in all the three FP cavities. In this configuration, by varying the position of $\mathrm{R}_{1}$ tuning of both the external cavities is accomplished.
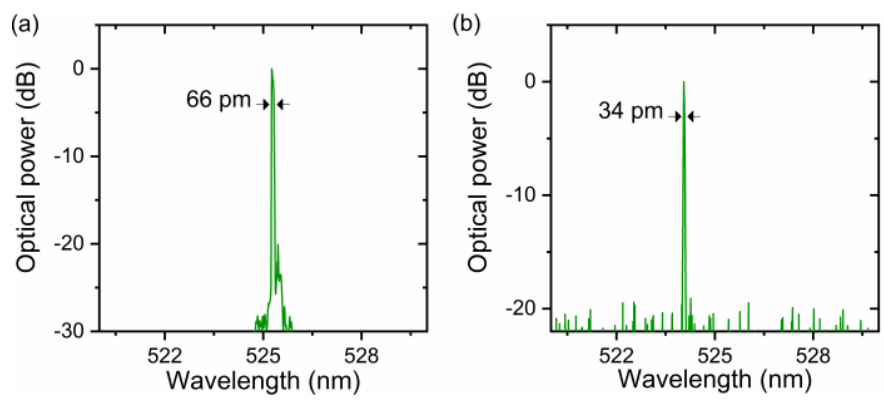

Fig. 4 Normalized optical lasing spectrums of single longitudinal mode with a very narrow linewidth of (a) 66 and (b) $34 \mathrm{pm}$, respectively. These values were recorded at $36 \mathrm{~mA}$ bias current and $20^{\circ} \mathrm{C}$ temperature.

The threshold current in a self-injection locked laser diode reduces from its free running counterpart - a clear sign of locking phenomenon [24]. By executing a two-stage selfinjection locking just below the threshold of the free running laser, at $36 \mathrm{~mA}$, and $20^{\circ} \mathrm{C}$ temperature, a narrow locked mode linewidth of $\sim 66 \mathrm{pm}$ at $525.26 \mathrm{~nm}$ is obtained and depicted in Fig. 4 (a). Furthermore, by careful and fine tuning of external cavity lengths, a linewidth of $\sim 34 \mathrm{pm}$ at $524.05 \mathrm{~nm}$ is measured, as shown in Fig. 4 (b). The latter measured value is two times narrower than the linewidth obtained in the tunable laser system employing single-stage self-injection locking. It is noteworthy to mention that the measured linewidth of $\sim 34 \mathrm{pm}$ is smaller than the laser diode mode spacing of $\sim 60 \mathrm{pm}$ suggesting a near single frequency operation. Besides, the measured linewidth is found to be near the resolution limit of the OSA $(\sim 20 \mathrm{pm})$, indicating a possibility of even narrower linewidth achievement by the system. Lastly, we noted that the stability of this two-stage self-injection locking system is comparatively inferior to that of the single-stage self-injection locking system of Fig. 1(a), which has been utilized for tunable as well as multi-wavelength generation. This is expected since fulfilling the stringent phase condition requirement with the manual tuning of this three-cavity system is non-trivial. 
Deployment of a piezo controlled tuning stage should enable achievement of stable operation from this system.

\section{Multi-WAVELENGTH LASER}

\section{A. Dual Longitudinal Wavelength Generation}

Employing the ECDL system shown in Fig. 1 (a) with singlestage self-injection locking, we were able to lock two longitudinal modes simultaneously; a highly attractive scheme in microwave photonics for the optical generation of the millimeter and terahertz beat frequencies (i.e., the frequency difference between the two modes). Fig. 5(a) shows the two color locked spectrum with a mode spacing of $\sim 0.6 \mathrm{~nm}$, corresponding to $\sim 653 \mathrm{GHz}$ beat frequency, obtained at 36 $\mathrm{mA}$ injection current. Moreover, at the same biasing condition, on the further slight tuning of the external cavity length, the beat frequency could be altered to $\sim 642, \sim 662 \mathrm{GHz}$ and even higher values; a result of the change in the wavelength spacing between the two modes. These results are promising for tunable $\mathrm{THz}$ frequency wave generation in the optical domain. Moreover, in Fig. 5 (b), the peak power ratio, which is the ratio of the peak powers of the two locked longitudinal modes, exhibited a value $<0.6 \mathrm{~dB}$ implying almost equal peak as well as integrated power between the modes since a comparable modes linewidth of $<200 \mathrm{pm}$ is also observed. Furthermore, the peak to ASE ratio for both the modes is $\sim 18 \mathrm{~dB}$, an essential requirement for high-quality $\mathrm{THz}$ frequency generation. Observe that the usable optical power (after $R_{2}$ ) in this case is small owing to just above threshold current operation. Nonetheless, the optical power of the system could be improved significantly by biasing the laser diode at high current injection and employing high power watt level InGaN/GaN laser diode since dual locking is found to be nearly independent of operating current.
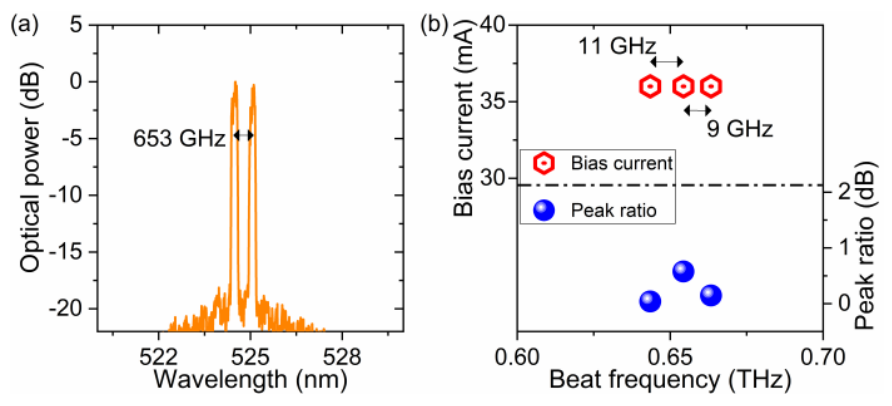

Fig. 5 (a) Normalized dual longitudinal mode operation generating $653 \mathrm{GHz}$ of beat frequency and (b) beat frequencies generated at $36 \mathrm{~mA}$ bias current and the respective peak ratio of the modes.

\section{B. Multiple Longitudinal Wavelength Generation}

By further careful tuning of the external cavity length, strikingly, the self-injection locked ECDL system (Fig. 1 (a)) allowed simultaneous locking of 1 to 4 modes, as plotted in Fig. 6. This accomplishment ascertains that multiple frequency modes, which are comparatively closely spaced, could be phase-matched and locked simultaneously in the two-cavity system. Also, these multiple wavelength spectra displayed in Fig. 6, correspond to different injection currents and temperatures. For instance, multi-wavelength spectrums with 3 simultaneous locked FP modes is achieved at $37 \mathrm{~mA}$ bias current and $20^{\circ} \mathrm{C}$, whereas 4 such locked modes spectrum is obtained at $50 \mathrm{~mA}$ injection and $40^{\circ} \mathrm{C}$ temperature. As discussed above, dual and even multi-wavelength locking is observed at all injection current and temperatures (independent of the diode laser operating conditions), so we selectively optimized few of them and presented in Fig. 6. This remarkable feature of our system not only assists in usable power enhancement but also indicates the flexibility and robustness of the configuration. Moreover, injection current and temperature could also serve as varying parameters in addition to the external cavity length, thereby offering an extra degree of freedom for simultaneous generation of several selfinjection locked FP modes with uniform wavelength spacing and hence exhibiting multiple wavelength lasing characteristics.
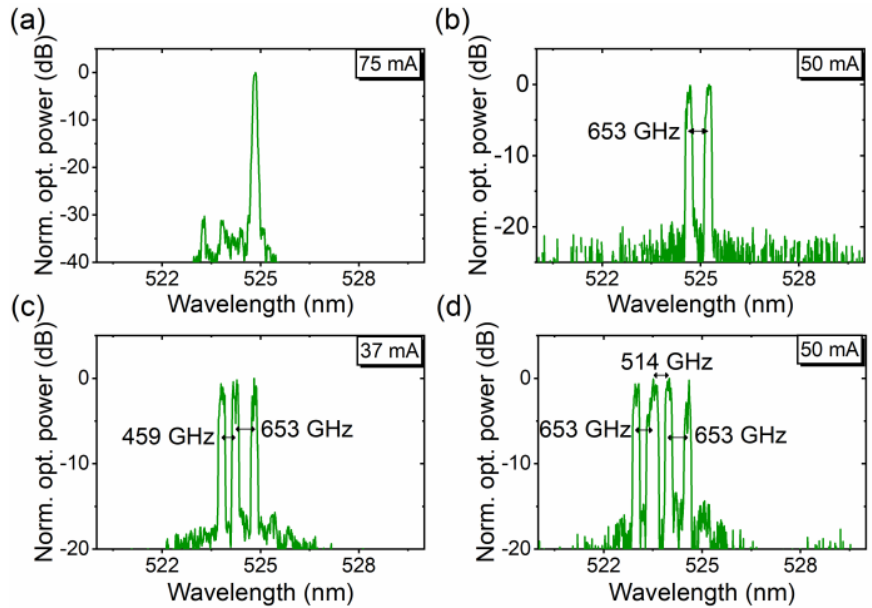

Fig. 6 Normalized multi-wavelength spectrums of self-injection locked external cavity system with simultaneous (a) single, (b) dual, (c) 3, and (d) 4 mode generation, at an injection current (temperature) of $75 \mathrm{~mA}\left(20^{\circ} \mathrm{C}\right), 50$ $\mathrm{mA}\left(40^{\circ} \mathrm{C}\right), 37 \mathrm{~mA}\left(20^{\circ} \mathrm{C}\right)$, and $50 \mathrm{~mA}\left(40^{\circ} \mathrm{C}\right)$, respectively.

Next, we analyze the performance of the multi-wavelength emission, firstly, by plotting in Fig. 7(a), the normalized integrated power distribution (in \%) of each locked mode of Fig. 6 by neglecting the ASE noise. Evidently, 100\% of the optical power in_Fig. 6 (a) is encompassed in the single locked mode while the power is almost equally distributed in dual lasing wavelength case of Fig. 6 (b), which is estimated to be $\sim 5.3 \mathrm{~mW}$ per mode and $\sim 10.3 \mathrm{~mW}$ in total, measured at the output of $R_{1}$. In this case, the shorter and longer wavelength mode constitutes 54 and $46 \%$ of the total integrated power, respectively, with a corresponding minimal standard deviation of 0.01, as can be seen from Fig. 7 (a) and (b). Considering the 3 frequency comb line spectrum, again a uniform $\sim 30 \%$ power distribution is observed across each mode with a uniform standard deviation of 0.01 . However, this value increased to 0.04 for the comb source with 4 locked FP modes, suggesting a larger variation of the power distribution among the modes. Fig. 7 (a) shows this uneven power distribution, with mode 2 and mode 4 exhibiting 37 and $16 \%$ of the total power, respectively. This is also reflected by the broad linewidth 
exhibited by mode $2(\sim 240 \mathrm{pm})$ compared to other modes (mode 1: $180 \mathrm{pm}$, mode 3: $190 \mathrm{pm}$, and mode 4: $\sim 120 \mathrm{pm}$ ), as illustrated in Fig. 7(c). We postulate that in the 4 locked modes lasing spectrum, the complete gain profile might have been occupied by the longitudinal modes with lower power distributed to the two extreme modes (i.e., 1 and 4), and hence resulting in smaller linewidth due to better localized injection ratio of these modes. On the other hand, the modes occupying the central part of the gain profile (i.e., 2 and 3) probably exhibits comparatively inferior localized injection ratio and hence meager locking efficiency, as displayed by their wider linewidths. In general, the spectral linewidths of each locked mode of Fig. 6 (a), (b), and (c) multi-wavelength spectrums are found to be similar exhibiting mean values of $\sim 100, \sim 130$, and $\sim 170$ pm, respectively, as shown in Fig. 7 (c). This noticeable increase in mode linewidth with the number of locked modes appearing in the spectrum is possibly to compensate for the composite phase matching condition of each longitudinal modes simultaneously.

Another essential characteristic of a multi-wavelength laser is the requirement of a uniform free spectral range (FSR) or mode spacing across all the modes. This is also investigated in Fig. 7(c) by plotting the mode spacing variation across adjacent locked modes for all the wavelength spectrums of Fig. 6. Note that the mode spacing is measured as the difference between the central lasing wavelengths (calculated at the fullwidth-at-half-maximum) of the neighboring modes. A mode variation of $\sim 0.52 \pm 0.08 \mathrm{~nm}(583 \pm 70 \mathrm{GHz})$ is measured for the 4 comb lines source while the corresponding value for the 3 and 2 locked mode spectrums are $\sim 0.5 \pm 0.1 \mathrm{~nm}(551 \pm 92$ $\mathrm{GHz}) \quad$ and $\sim 0.6 \mathrm{~nm}(653 \mathrm{GHz})$, respectively, thus preserving the characteristics of multi-wavelength generation with appreciably small FSR variation. We strongly believe that proper optimization of the system, particularly via the deployment of piezo stages for external cavity tuning; an equidistant mode spacing with comparable mode linewidth multi-wavelength generation is feasible.

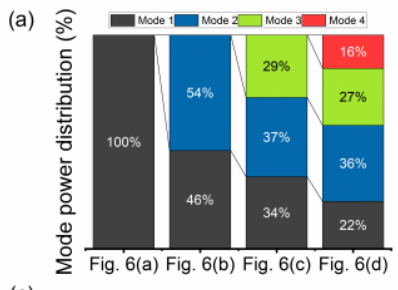

(c)
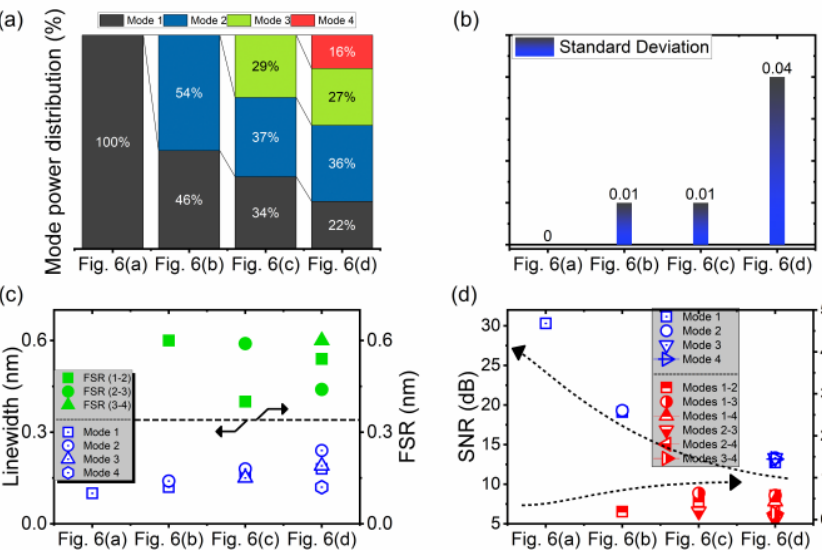

(d)

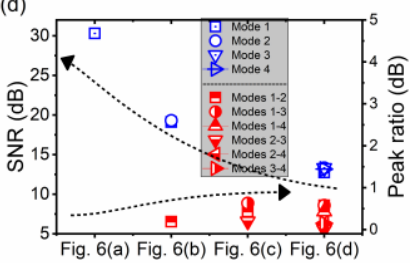

Fig. 7 (a) Optical power distribution, (b) standard deviation of the power distribution, (c) linewidth and FSR, and (d) SNR and peak power ratio of the individual longitudinal modes of various multi-wavelength spectrums of Fig. 6. The arrows in (c) and (d) are the guide to the eyes.
Next, we investigated the variation of SNR (ratio of the peak power of each mode to the ASE noise floor) and the peak power ratio across different multi-wavelength lasing emission of Fig. 6, and summarized the results in Fig. 7(d). The SNR is found to degrade as the number of modes in the multiwavelength laser increases. For instance, the SNR for a single mode spectrum is measured to be $\sim 30 \mathrm{~dB}$ at $75 \mathrm{~mA}$, attributed to the superior phase matching condition of the locked mode. This value subsequently decreased to $\sim 20 \mathrm{~dB}$ for the dual locked case and eventually to $\sim 13 \mathrm{~dB}$ for the 4 locked FP mode spectrum at $40^{\circ} \mathrm{C}$ and $50 \mathrm{~mA}$ bias. This is ascribed, partly to a similar integrated power $(\sim 10.3 \mathrm{~mW})$ distribution among the four modes in the latter case compared to the former dual modes case, as displayed in Fig. 7(a), and to the phase matching condition, which is quite rigorous in the latter case. On the other hand, peak power ratios are found to exhibit very close to $0 \mathrm{~dB}$ values in all the multi-wavelength spectrums suggesting almost equal peak powers across the modes of the spectrums. The minimum and maximum peak power ratios were found to be $\sim 0$ and $\sim 0.6 \mathrm{~dB}$, found between mode 2-3 and mode 1-3 in Fig. 6 (d) and Fig. 6 (c), respectively. This again affirms the quality of the generated multi-wavelength spectrums with flat-top and high SNR that is crucial in applications like WDM-VLC since any amplification would result in a consistent power levels across the modes thus negating incorporation of gain-flatting filters in the communication system.
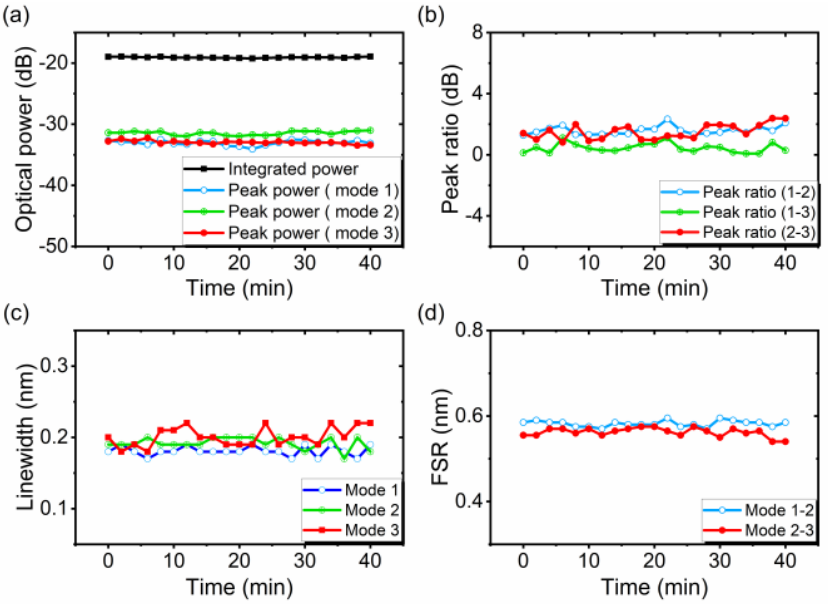

Fig. 8 Stability test of the multi-wavelength laser emission with 3 simultaneously locked modes, showing the time variation of (a) peak power of each mode and the total integrated power (arb. units), (b) peak power ratio across the modes, (c) linewidth of the modes, and (d) FSR across the modes.

\section{Stability Analysis}

Lastly, we analyzed the stability of the generated multiple wavelength FP modes to further support the potential deployment of the proposed self-injection locked based ECDL system in practical applications. Accordingly, we performed a short-term stability test on the 3 locked mode spectrum using the experimental setup shown in Fig. 1(a), where the optical powers, peak ratios, linewidths, and FSRs have been recorded at every 2 minutes time interval for over 40 minutes. The 
results are summarized in Fig. 8 with laser diode biased at 43 $\mathrm{mA}$ and operated at $20^{\circ} \mathrm{C}$. A variation of $\pm 0.8 \mathrm{~dB}$ in the peak power of mode 1 is observed from Fig. 8(a) whereas the other modes were found to be very stable. Hence, the total integrated power of the system remained almost constant, which is vital in several applications. Fig. 8(b) and (c) shows minor fluctuations in the peak power ratio and mode linewidth across the three locked wavelength modes over time depicting a random trend, which suggests a slightly noisy system. Proper optimization of the system further (i.e., external cavity length) should decrease these variations and hence the noise of the system considerably. Nevertheless, a variation of the peak power ratio across modes $1-2,1-3$, and 2-3 are $\pm 0.5 \mathrm{~dB}, \pm 0.5$ $\mathrm{dB}$ and $\pm 0.7 \mathrm{~dB}$, respectively, and the linewidth fluctuation of mode 1,2 , and 3 are measured to be $\pm 10 \mathrm{pm}, \pm 15 \mathrm{pm}$, and \pm 20 pm. This suggests that modes 1 and 2 are more stable than mode 3 in general, which is further reflected in Fig. 8(d) that shows the variation in FSR across the three locked modes. While the variation of $\pm 10 \mathrm{pm}$ is observed between modes 1 2 , modes 2-3 exhibited a corresponding value of $\pm 20 \mathrm{pm}$. Nonetheless, overall, the system is found to be very stable, which indicates that the generated multi-wavelength emission qualifies as potential sub-carriers in WDM-VLC.

\section{CONCLUSION}

To summarize, a single and two stage self-injection locking ECDL arrangement with an $\mathrm{InGaN} / \mathrm{GaN}$ green laser diode is developed to demonstrate a multi-wavelength and near single wavelength generation at $\sim 525 \mathrm{~nm}$. Successful simultaneous locking of one to four FP modes with $\sim 0.5 \mathrm{~nm}$ FSR and up to $\sim 30 \mathrm{~dB}$ SNR is demonstrated. This first-of-its-kind visible multi-wavelength laser system exhibited mean $\sim 150 \mathrm{pm}$ mode linewidth with similar peak and integrated power levels, and appreciably stable, particularly in terms of FP modes wavelength shift. Besides, an ultra-narrow near single wavelength linewidth of $\sim 34 \mathrm{pm}$ is measured from two stage self-injection locked ECDL system with $\sim 20 \mathrm{~dB}$ SMSR. Our proposed schemes are compact, simple and cost-effective compared to other ECDL configurations, and in particular, power efficiency since a usable optical power as high as $\sim 65 \%$ of the diode laser power is achieved, thus addressing a plethora of multi-disciplinary field applications that require coherent single and multiple wavelength visible laser sources.

\section{REFERENCES}

[1] R. Ana and M. Lopez-Amo, "Multi-Wavelength Fiber Lasers," in Current Developments in Optical Fiber Technology, S. W. Harun, Ed. InTech, 2013.

[2] T. J. Slight et al., "Continuous-wave operation of (Al,In)GaN distributed-feedback laser diodes with high-order notched gratings," Applied Physics Express, vol. 11, no. 11, p. 112701, Nov. 2018.

[3] Y. Chen, W. Lin, H. Chen, J. Shy, and H. Chui, "Single-Frequency External Cavity Green Diode Laser," IEEE Photonics Journal, vol. 9, no. 6, pp. 1-7, Dec. 2017.

[4] Y.-H. Chen, W.-C. Lin, J.-T. Shy, and H.-C. Chui, "Iodine-stabilized single-frequency green InGaN diode laser," Opt. Lett., OL, vol. 43, no. 1, pp. 126-129, Jan. 2018

[5] M. Chi, O. B. Jensen, and P. M. Petersen, "Green high-power tunable external-cavity GaN diode laser at 515 nm," Opt. Lett., OL, vol. 41, no. 18 , pp. 4154-4157, Sep. 2016.
[6] C. J. Hawthorn, K. P. Weber, and R. E. Scholten, "Littrow configuration tunable external cavity diode laser with fixed direction output beam," Review of Scientific Instruments, vol. 72, no. 12, pp. 4477-4479, Nov. 2001

[7] A. Rolland, P. Li, M. Cassinerio, J. Jiang, and M. Fermann, "Precision visible comb from an Er fiber laser," in Conference on Lasers and Electro-Optics (2018), paper JTh2A.185, 2018, p. JTh2A.185.

[8] Y. Yang et al., "Four-wave mixing parametric oscillation and frequency comb generation at visible wavelengths in a silica microbubble resonator," Opt. Lett., OL, vol. 41, no. 22, pp. 52665269, Nov. 2016.

[9] C.-Y. Lin, Y.-C. Chi, C.-T. Tsai, H.-Y. Chen, and G.-R. Lin, "Twocolor laser diode for 54-Gb/s fiber-wired and 16-Gb/s MMW wireless OFDM transmissions," Photonics Research, vol. 5, no. 4, p. 271, Aug. 2017.

[10] H.-Y. Wang, Y.-C. Chi, and G.-R. Lin, "Dual-mode laser diode carrier with orthogonal polarization and single-mode modulation for remote-node heterodyne MMW-RoF," Optics Letters, vol. 41, no. 20, p. 4676 , Oct. 2016.

[11] G.-R. Lin et al., "Long-Cavity Fabry-Perot Laser Amplifier Transmitter With Enhanced Injection-Locking Bandwidth for WDMPON Application," Journal of Lightwave Technology, vol. 28, no. 20, pp. 2925-2932, Oct. 2010.

[12] Gong-Ru Lin, Hai-Lin Wang, Gong-Cheng Lin, Yin-Hsun Huang, YiHung Lin, and Tzu-Kang Cheng, "Comparison on Injection-Locked Fabry-Perot Laser Diode With Front-Facet Reflectivity of $1 \%$ and $30 \%$ for Optical Data Transmission in WDM-PON System," Journal of Lightwave Technology, vol. 27, no. 14, pp. 2779-2785, Jul. 2009.

[13] L. Fan, G. Xia, J. Chen, X. Tang, Q. Liang, and Z. Wu, "High-purity $60 \mathrm{GHz}$ band millimeter-wave generation based on optically injected semiconductor laser under subharmonic microwave modulation," Optics Express, vol. 24, no. 16, p. 18252, Aug. 2016.

[14] M. Pochet, N. A. Naderi, Y. Li, V. Kovanis, and L. F. Lester, "Tunable Photonic Oscillators Using Optically Injected QuantumDash Diode Lasers," IEEE Photonics Technology Letters, vol. 22, no. 11, pp. 763-765, Jun. 2010.

[15] M. A. Shemis et al., "Broadly Tunable Self-injection Locked InAs/InP Quantum-dash Laser Based Fiber/FSO/Hybrid Fiber-FSO Communication at $1610 \mathrm{~nm}$," IEEE Photonics Journal, vol. 10, no. 2, pp. 1-10, Apr. 2018.

[16] M. A. Shemis et al., "L-Band Quantum-dash Self-Injection Locked Multiwavelength Laser Source for Future WDM Access Networks," IEEE Photonics Journal, vol. 9, no. 5, pp. 1-7, Oct. 2017.

[17] A. Hurtado, R. Raghunathan, I. D. Henning, M. J. Adams, and L. F. Lester, "Simultaneous Microwave- and Millimeter-Wave Signal Generation With a 1310-nm Quantum-Dot-Distributed Feedback Laser," IEEE Journal of Selected Topics in Quantum Electronics, vol. 21, no. 6, pp. 568-574, Nov. 2015.

[18] A. Hurtado, J. Mee, M. Nami, I. D. Henning, M. J. Adams, and L. F. Lester, "Tunable microwave signal generator with an opticallyinjected 1310nm QD-DFB laser," Optics Express, vol. 21, no. 9, p. 10772, May 2013.

[19] B. Janjua et al., "Going beyond 4 Gbps data rate by employing RGB laser diodes for visible light communication," Opt. Express, $O E$, vol. 23, no. 14, pp. 18746-18753, Jul. 2015.

[20] A. Sheetal and H. Singh, "5 $\times 10$ Gbps WDM-CAP-PON based on frequency comb using OFDM with blue LD," Opt Quant Electron, vol. 50, no. 12, p. 446, Nov. 2018.

[21] S. H. Younus and J. M. H. Elmirghani, "WDM for high-speed indoor visible light communication system," in 2017 19th International Conference on Transparent Optical Networks (ICTON), 2017, pp. 16.

[22] M. Kong et al., "10-m 9.51-Gb/s RGB laser diodes-based WDM underwater wireless optical communication," Opt. Express, OE, vol. 25, no. 17, pp. 20829-20834, Aug. 2017.

[23] M. H. M. Shamim et al., "Investigation of Self-Injection Locked Visible Laser Diodes for High Bit-Rate Visible Light Communication," IEEE Photonics Journal, vol. 10, no. 4, pp. 1-11, Aug. 2018. 
[24] M. H. M. Shamim, T. K. Ng, B. S. Ooi, and M. Z. M. Khan, "Tunable self-injection locked green laser diode," Opt. Lett., $O L$, vol. 43, no. 20, pp. 4931-4934, Oct. 2018.

[25] C. M. Schultz, "Analysis and mitigation of the factors limiting the efficiency of high power distributed feedback diode lasers," Technische Universität Berlin, 2013.

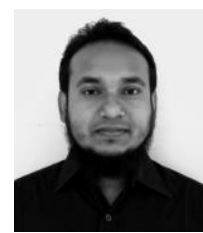

Md Hosne Mobarok Shamim received the B.Sc. degree in Electrical and Electronic Engineering from Islamic University of Technology, Dhaka, Bangladesh in 2013. Later, he graduated from King Fahd University of Petroleum \& Minerals, Dhahran, Saudi Arabia with a M.Sc. degree in 2018. Currently, he is pursuing his Ph.D. at McGill University, Canada as a recipient of McGill Engineering Doctoral Award. His research interest includes semiconductor lasers and supercontinuum sources.

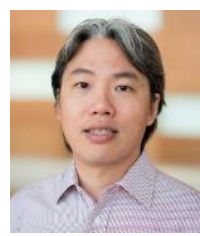

Tien Khee Ng (Senior Member of IEEE, Member of SPIE, and Senior Member of OSA) received his Ph.D. (2005) and M.Eng. (2001) from Nanyang Technological University (NTU), Singapore. He was a Test Engineer at Hewlett-Packard Singapore (1997-1998), a member-oftechnical-staff with Tinggi Technologies (2004-2006), and a research fellow at NTU until 2009. He is a senior research scientist with Ooi-group at King Abdullah University of Science and Technology (KAUST), Saudi Arabia. As a co-principal-investigator responsible for innovation in MBEgrown nanostructures devices and in laser devices, at the KACST Technology-Innovation-Center at KAUST, he realized wide-bandgap nitride quantum-confined and nanowires structures for light-emitters technology, optical wireless communications, and energy harvesting.

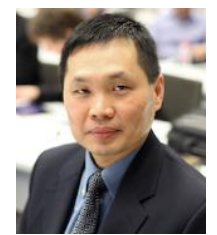

Boon S. Ooi is a Professor of Electrical Engineering at King Abdullah University of Science and Technology (KAUST), Twual, Saudi Arabia. He received the Ph.D. degree from the University of Glasgow (UK) in 1994. He joined KAUST from Lehigh University (USA) in 2009. His recent research is concerned with the study of IIINitride based materials and devices, and lasers for applications such as solid-state lighting, visible light and underwater wireless optical communications, and energy harvesting devices. He has served on the technical program committee of CLEO, IPC, ISLC and IEDM. He currently serves on the editorial board of Optics Express and IEEE Photonics Journal. He is a Fellow of OSA, SPIE and IoP (UK).

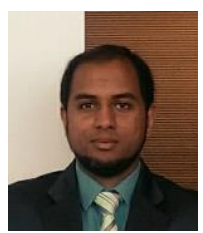

Mohammed Zahed M. Khan received B.E. degree in electronics and communication from Osmania University, India, in 2001, M.S. degree in electrical engineering from King Fahd University of Petroleum and Minerals (KFUPM), Saudi Arabia, in 2004, and PhD degree in electrical engineering from King Abdullah University of Science and Technology (KAUST), Saudi Arabia, in 2013. Prior to joining KFUPM, he was a SABIC postdoctoral research fellow in Photonics Laboratory, KAUST, from 2014-2015. His prior research involved developing numerical models for integrated optical device simulation. Currently, his research focus is on development of near infrared and visible semiconductor lasers and systems for applications in optical communications. He is a senior member of IEEE. 